\title{
Teaching Tools Made With Excel Spreadsheets to Study the Isothermal Process in an Ideal Gas
}

\author{
Ionel Grigore \\ High School Nichita Stanescu, Ploiesti, Romania \\ Gheorghe Dumitrescu \\ High School Toma N. Socolescu, Ploiesti, Romania \\ Daniela Stoica \\ High School Ion Luca Caragiale, Ploiesti, Romania
}

\begin{abstract}
This paper demonstrates how Excel spreadsheets can be explored in order to analyze the isothermal process in an ideal gas. It describes three tools made with spreadsheets to facilitate students' understanding of the model of the ideal gas and the isothermal transformation. With the help of the first tool we can draw a comparison between the theoretical model which describes the isothermal process for an ideal gas and the experimental data in order to verify the Boyle-Mariotte law. With the help of the second tool we can analyze the isothermal process in an ideal gas by the graphic representation of the isotherm curve in the pressure-volume coordinates. This tool can also calculate, both analytically and numerically, the work exchanged by the gas with the external environment. The numerical calculation of the work is based on the geometrical interpretation of this measure in the pressure-volume coordinates. The third tool provides the comparison between the isothermal process and the adiabatic process in an ideal gas when both processes are characterized by the same initial state. The classroom utilization of these three tools develops students' skills in Excel for data manipulation to calculate certain thermodynamic measures and leads to the clarification of certain fundamentals concepts of thermodynamics, such as the isothermal transformation and the work. The numerical calculation of the work with the help of the facilities offered by the spreadsheet contributes to students' understanding of a key concept of definite integral calculus.
\end{abstract}

Keywords: spreadsheets, isothermal transformation, Boyle-Mariotte law, work in thermodynamics, educational physics

\section{Introduction}

The teaching and learning of thermodynamics has a significant place among research topics in educational physics. The articles in the literature on this subject analyze the difficulties faced by students in the study of thermodynamics and propose various remedies to effectively address this area of physics.

Thus, investigating students' understanding of the first principle of thermodynamics has shown that they do not manage to frequently discern the differences between the concepts of heat, temperature, work, and internal energy. The authors suggest that the misinterpretation of simple microscopic patterns can affect the

Ionel Grigore, Ph.D., teacher, High School Nichita Stanescu.

Gheorghe Dumitrescu, Ph.D., teacher, High School Toma N. Socolescu.

Daniela Stoica, Ph.D., teacher, High School Ion Luca Caragiale. 
comprehension of macroscopic phenomena (Loverude, Kautz, \& Heron, 2002). An integrated course and laboratory module have been developed on the behaviour of gases in order to engage students in the process of scientific discovery. The topic of gases has represented the medium employed to illustrate important research aspects in Physics, namely, the processing of experimental data or the relation between experimental data and scientific models. Thus, students measure the macrosocopic properties of a gas in the laboratory, put the data together in a mathematical relation between the variables, and use this relation to develop a model of microscopic behaviour (Clarkson, Mills, Sweeney, \& Marino, 2000). For the study of the simple transformations of the ideal gas and for the analysis of the basic thermodynamics cycles applications have been developed in the $\mathrm{C}$ \# language, so that students can have a better grasp of the principles underlying thermodynamics and the way in which these principles can be used to solve problems (Liu, 2011).

\section{Literature Review}

In solving thermodynamics problems, various authors have promoted spreadsheets as tools that can be developed by students, instead of speacialized programmes. The emphasis was firstly placed on the understanding of the fundamental idea to solve the problem and less on the technique to solve the equation of the problem (Sandler, 1997). Spreadsheets have been used to simulate Boyle's law to allow students to have a better grasp of a simple thermodynamics experiment. It was showed how calculation errors can be taken into consideration and how they influence the final results. With the help of spreadsheets teachers can unitarily explore numerical, graphical, symbolic, and verbal aspects and can properly modify spreadsheets to satisfy students' needs (Sinex, 2008). In the same context, there have been generated models of thermodynamics systems demonstrating the effects of the changes in the input parameters on the final results (Caretto, McDaniel, \& Mincer, 2005) and it has been shown how Excel spreadsheets can be used to calculate the thermal balance for diverse substances known by minimizing the Gibbs energy. The results have been compared to those obtained by using a programme written in FORTRAN language and a satisfactory concordance has been found (Lwin, 2000). Using spreadsheets helps students efficiently fulfil the objectives in the physics laboratory by easily processing the sets of experimental data and by assessing the complex analytic and numeric models without requiring special programming skills (Stammitti, 2013).

This paper describes three teaching tools made with the help of Excel spreadsheets for the teaching and learning of concepts specific to thermodynamics from the subject of the isothermal transformation of the ideal gas. The classroom use of each tool can clarify different aspects concerning the isothermal transformation of the ideal gas.

The first tool allows the comparison between the theoretic model of an isothermal process in an ideal gas and the data resulted from the experiment. With this tool, we can easily process data from a didactic experiment where we verify the Boyle-Mariotte law, analyzing the isothermal compression of the air from a $U$-shaped glass, following the processing of the experimental data, using the equation of the isothermal transformation under a linearized form, and the atmospheric pressure from the laboratory can be determined.

The second tool facilitates the analysis of the isothermal process in an ideal gas by the graphic representation of the isothermal curve in the pressure-volume coordinates highlighting the initial and final states of the process. The isothermal curve corresponding to the value of the temperature from the input data overlaps the isothermal curve corresponding to the temperature $t_{0}=0{ }^{\circ} \mathrm{C}$. By changing the input data, we can trace how the isothermal curve moves in relation to the origin of the system of coordinates and to the 
isothermal corresponding to the temperature $t_{0}=0{ }^{\circ} \mathrm{C}$. Also, this tool facilitates the calculation of the work exchanged by the gas with the external environment in the isothermal process. The calculus relation of the work in the isothermal process is verified utilizing the geometric interpretation of this measure within the pressure-volume graph. Thus, we compare the value of the work calculated analytically to the value of the work calculated numerically with the trapezoidal method in the evaluation of a definite integral.

The third tool offers a comparative analysis between an isothermal process and an adiabatic process characterized by the same initial state. By using this tool, we can observe the difference between the isothermal curve and the adiabatic curve in the pressure-volume coordinates and we can analyze how these two curves change one in relation to the other when modifying the input data.

\section{Organization of Spreadsheets}

Each tool described in this paper is made up of several interconnected Excel spreadsheets. The results obtained, presented both analytically and graphically, are placed next to the input data, so that the feedback to data change can be easily traced. The tools presented in other papers have a similar structure, presenting the facilities of Excel spreadsheets in the process of teaching-learning of physics (Grigore, Miron, \& Barna, 2014; 2015a).

The first Excel tool has been developed for the processing of data in a didactic experiment to verify the Boyle-Mariotte law. This experiment can be performed in various forms, some of which are largely described in the literature (Argesanu, 2011; Lewis, 1997; Richmond, 1997). Next, we present the basic principle to conduct the experiment and the way in which we processed the data with spreadsheets. To perform the experiment, we use a $U$-shaped glass tube closed at one end and open at the other. We pour water in the open branch of the tube and the air from the closed branch is compressed. This creates a dishevelment $h$ between the levels of the water from the two branches. Noting the atmospheric pressure $p_{0}, \rho=$ "Density of water," $g=$ "Gravitational acceleration," $S=$ "Area of the transversal section of the closed tube," and $L=$ "The length of the air column from the tube," then the air pressure and volume are given by the relations:

$$
\begin{gathered}
p=p_{0}+\rho g h \\
V=S L
\end{gathered}
$$

Approximating the air from the tube with an ideal gas and considering the law of the isothermal transformation we can write:

$$
\left(p_{0}+\rho g h\right) S L=K
$$

where $\mathrm{K}$ is a constant.

Introducing the notation:

$$
C=\frac{K}{\rho g S}
$$

we can rewrite equation (3) under the form:

$$
h L=C-\frac{p_{0}}{\rho g} L
$$


Consequently, according to Equation (5), measuring $h$ and $L$, we can verify the Boyle-Mariotte law if the graph of the product $h L$ in relation to $L$ represents a straight line. From the slope of this straight line, we can determine the atmospheric pressure $p_{0}$ (Argesanu, 2011). The value of the atmospheric pressure calculated after data processing can be compared to the value of the pressure measured with the barometer in the laboratory.

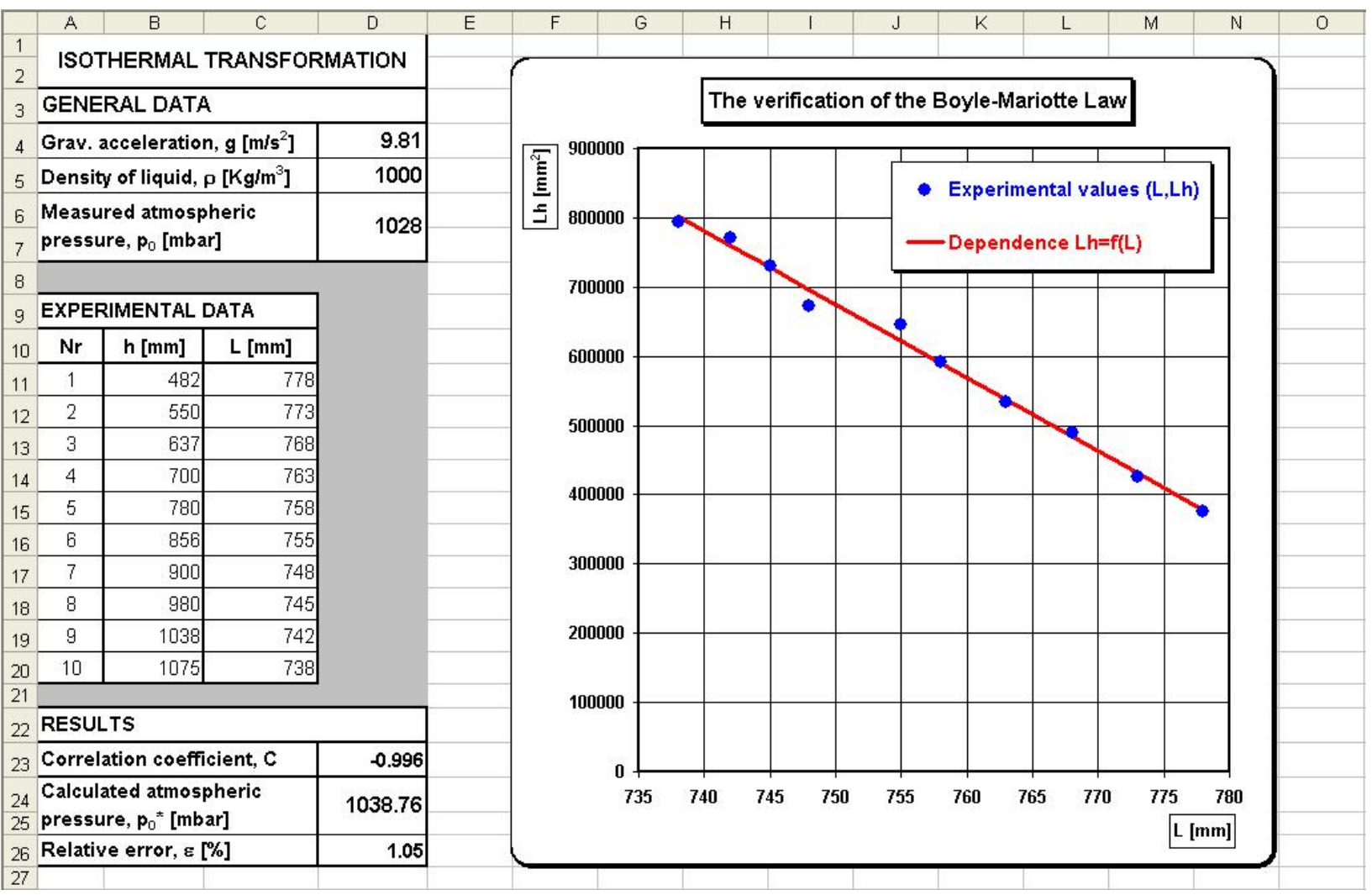

Figure 1. Main spreadsheet of the tool intended for the verification of the Boyle-Mariotte law.

\begin{tabular}{|c|c|c|c|c|c|c|c|c|}
\hline & A & $B$ & C & D & $E$ & $\mathrm{~F}$ & G & $\mathrm{H}$ \\
\hline 1 & \multirow{2}{*}{\multicolumn{5}{|c|}{ SOURCE TABLE FOR THE GRAPH }} & & & \\
\hline & & & & & & & & \\
\hline 3 & No. & $h[\mathrm{~mm}]$ & L [mm] & $\operatorname{Lh}\left[\mathrm{mm}^{2}\right]$ & $(\mathrm{Lh})^{*}\left[\mathrm{~mm}^{2}\right]$ & & & \\
\hline 4 & 1 & 482 & 778 & 374996 & 378300.34 & & & \\
\hline 5 & 2 & 550 & 773 & 425150 & 431244.32 & & & \\
\hline 6 & 3 & 637 & 768 & 489216 & 484188.29 & & & \\
\hline 7 & 4 & 700 & 763 & 534100 & 537132.27 & & & \\
\hline 8 & 5 & 780 & 758 & 591240 & 590076.25 & & & \\
\hline 9 & 6 & 856 & 755 & 646280 & 621842.63 & & & \\
\hline 10 & 7 & 900 & 748 & 673200 & 695964.20 & & & \\
\hline 11 & 8 & 980 & 745 & 730100 & 727730.58 & & & \\
\hline 12 & 9 & 1038 & 742 & 770196 & 759496.97 & & & \\
\hline 13. & 10 & 1075 & 738 & 793350 & 801852.15 & & & \\
\hline 14 & & & & & & & & \\
\hline 15 & & & & & & & & \\
\hline
\end{tabular}

Figure 2. The graph source table of the tool intended for the verification of the Boyle-Mariotte law.

Figure 1 renders the main spreadsheet of the tool. It can be observed that it has been divided into three sections plus the area of the associated graph. In the first section, entitled "General data" and limited by the domain A3: D6, we introduce the values for the following physical measures: gravitational acceleration, $g$, in 
cell D4; density of the liquid used, $\rho$, in this case the density of water, in cell D5; and the atmospheric pressure measured with the manometer in the laboratory, $p_{0}$, in cell D6. In the second section, entitled "Experimental data," limited by the domain A9: C20, we introduce, in columns B and C; the values obtained from measurements for the dislevelment of the water column, $h$; and the corresponding length of the air column, $L$. In the third section, entitled "Results," we calculate the correlation coefficient of the experimental data, after the linear regression, in cell D23, the atmospheric pressure in cell D24, and the relative error when calculating the atmospheric pressure in cell D26, following the comparison to the value measured for the pressure from cell D6. As observed in the Figure 1, the unit of measure is specified for each measure that appears in the spreadsheet.

The graphic results are presented in the graph associated to the tool and placed next to the tables of the three sections. The blue dots render the pairs of experimental values $(h, L h)$ and the red straight line represents the dependence of the product $L h$ in relation to the height $h$ following the linear regression of the experimental data. It can be observed that the graphic representation is in conformity with Equation (5).

The source table of the graph, placed in a secondary sheet, is rendered in Figure 2. This table comprises the domain A3: E13. In column A, we have the number of the measurement, and in columns B and C, there are imported from the main spreadsheet the measured values of the height of the water column dislevelment and of the air column length from the glass tube. In column D, we calculate the values of the composition of the height $h$ and the length $L$ from the previous columns; and in column E, we have the linear regression of the data from columns $\mathrm{C}$ and $\mathrm{D}$.

To perform the calculations in Excel, we have used the following names of cells in the main spreadsheet: "Acceleration_G" for cell D4, "Density_L" for cell D5, and "Pressure_A" for cell D6. In the secondary sheet where there is the source table of the graph we have used the names of domains "Length" for the domain C4: C13 and "Composition_LH" for the domain D4: D13.

To perform the linear regression in column E from the source table of the graph we have used the functions SLOPE and INTERCEPT to calculate the slope of the straight line respectively, the ordinate at the origin. Thus, with the help of the two functions, we have written in cell E4 the following Excel formula:

"= SLOPE (Composition_LH; Length)*C4 + INTERCEPT (Composition_LH; Length)".

The formula from cell E4 has been propagated in column E up to cell E13. By selecting the data from columns $\mathrm{C}, \mathrm{D}$, and $\mathrm{E}$, we made the graph from the main spreadsheet.

To calculate the correlation coefficient in cell D23 we have written the following Excel formula:

"CORREL (Length; Composition_LH)".

To calculate the atmospheric pressure in the main spreadsheet in cell D24 we have considered Equation (5) writing the following Excel formula:

“=Density_L*Acceleration_G*ABS (SLOPE (Composition_LH; Length))*10^(-5)”.

The multiplication by factor $10^{-5}$ in the above formula has been made to express the pressure in the units of measure specified in the table.

Further, we describe the second Excel tool to analyze the isothermal transformation of the ideal gas as a slightly modified version of the tool described in a previous paper of the author (Grigore, Miron, \& Barna, 2015b). The main spreadsheet of this tool is rendered in Figure 3. The section "Input data" comprises the domain A3: B10 and the section "Results" comprises the domain A12: B23.

In the section "Input data," we introduce the values for the following measures: the universal gas constant, 
$\mathrm{R}$, in cell $\mathrm{B} 4$, the molar mass, $\mu$, in cell $\mathrm{B} 5$, the mass of the gas, $m$, in cell $\mathrm{B} 6$, the temperature when the isothermal process occurs, $T$, in cell $\mathrm{B} 7$, the volume of the initial state, $V_{l}$, in cell $\mathrm{B} 9$, the volume of the final state, $V_{2}$, in cell $\mathrm{B} 10$. As for the previous tool, there are specified the units of measurement in the spreadsheet for each physical measure.

The section "Results" is divided into two subsections. In the first subsection, given by the domain A12: $\mathrm{B} 15$, we calculate the pressure of the gas in initial and final states, $p_{1}, p_{2}$, in cells B13, B14, and the work exchanged by the gas with the external environment, $L$, in cell B15. In the second subsection, given by the domain A17: B23, we calculate the work through the numerical method using the geometrical interpretation of this measure in $\mathrm{p}-\mathrm{V}$ coordinates. In cell $\mathrm{B} 19$, we introduce the number of divisions, $n$, in which we divide the interval $\left[V_{1}, V_{2}\right]$, in cell B20, we calculate the elementary interval $\delta V=\left(V_{2}-V_{l}\right) /(n-1)$, whereas in cell B21, we calculate the work, $L^{*}$, as the area under the isothermal curve corresponding to the interval $\left[V_{1}, V_{2}\right]$. The value of the work calculated numerically in cell B21 is compared to the value of the work calculated analytically in cell B15.

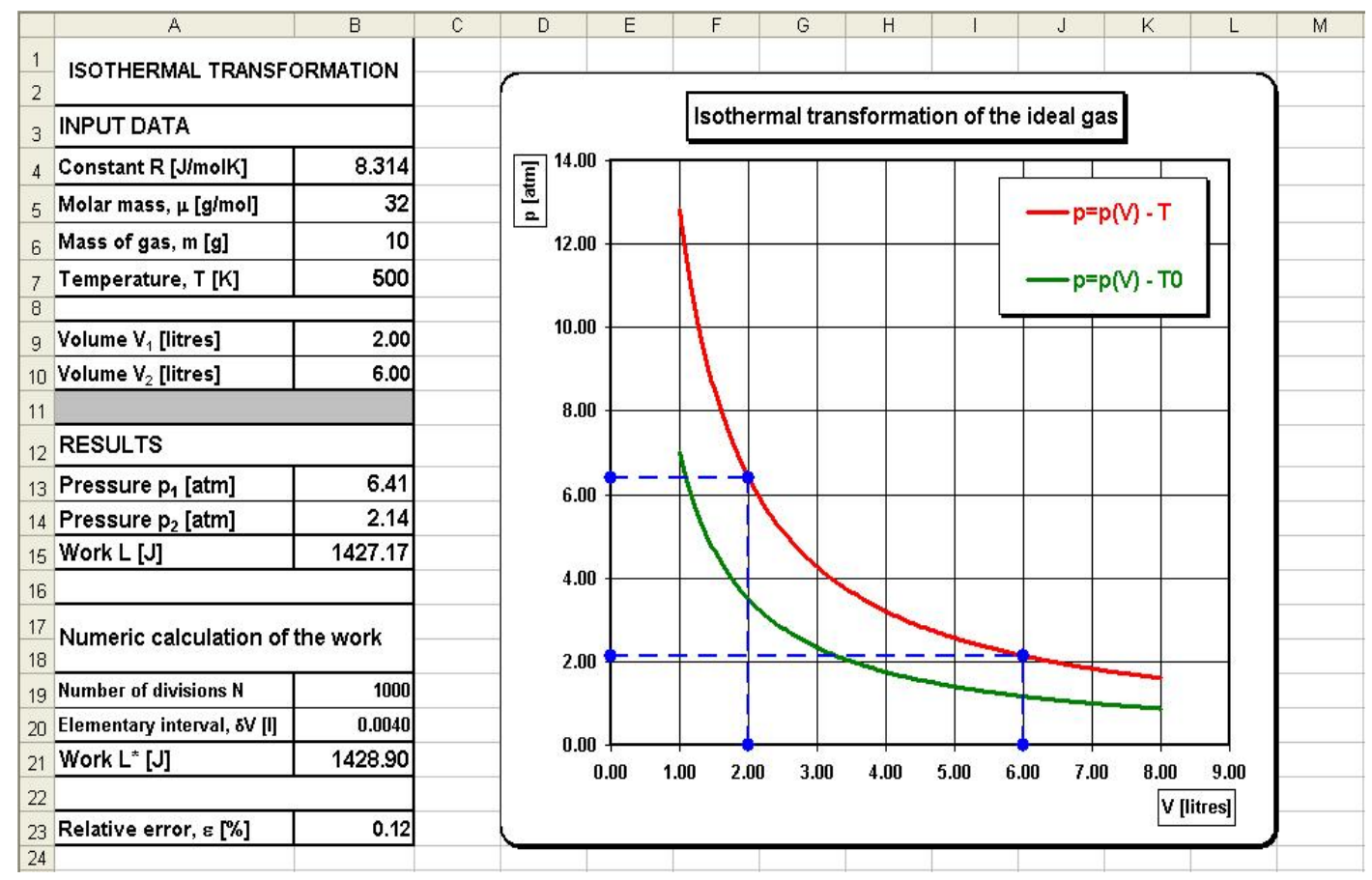

Figure 3. The main spreadsheet of the tool for the analysis of the isothermal transformation of the ideal gas.

To perform the calculations in Excel, we have introduced the following names of cells: "Constant_R" for cell B4, "Mass_M" for cell B5, "Mass_G” for cell B6, "Temperature" for cell B7, "Volume_1" for cell B9, and "Volume_2" for cell B10.

To calculate the pressure in the initial and final states we have used the equation of the thermal state of the ideal gas. Thus, in cells B13 and B14, we have written the following Excel formulas:

$"=(($ Mass_G/Mass_M $) *$ Constant_R $*$ Temperature $) /\left(\right.$ Volume_ $\left.1 * 10^{\wedge}(-3)\right) *(1 / 101325) " ;$

$"=(($ Mass_G/Mass_M $) *$ Constant_R*Temperature $) /($ Volume_2*10^(-3) $) *(1 / 101325) "$.

In the previous Excel formulas, we have considered the conversion of the units of measurement, so that the values of the pressure results in atmospheres (ATM) as specified in the spreadsheet. 
For the analytical calculation of the work we have used the relation (Serway \& Jewett, 2013):

$$
L=\int_{V_{1}}^{V_{2}} p_{(V)} d V=\frac{m}{\mu} R T \ln \left(\frac{V_{2}}{V_{1}}\right)
$$

Thus, in cell B15, we have written the following Excel formula:

“= (Mass_G/Mass_M)*Constant_R*Temperature*LN (Volume_2/Volume_1)".

The graph associated to the results from the main spreadsheet renders the isothermal curve of the process in pressure-volume coordinates through a continuous red line. The pairs of values pressure-volume corresponding to the initial and final states are highlighted by the segments traced in a dotted line colored in blue. Also, on the graph, there is represented the isothermal corresponding to the temperature $t_{0}=0{ }^{\circ} \mathrm{C}\left(T_{0}=\right.$ $273.15 \mathrm{~K}$ ) by a continuous green line in order to be compared to the isothermal corresponding to the temperature from the input data. The source table of the graph, placed in a secondary sheet, contains the column of the values of the volume and the columns in which we calculate the pressure for the isothermal process characterized by the temperature $T$ from the input data and for the temperature $T_{0}$. Also, we have used supplementary columns for the series of data highlighting the values of the pressure and volume for the initial and final states.

For the numerical calculation of the work, we have made another secondary sheet in the source table rendered partially, with the first 20 lines, as shown in Figure 4.

\begin{tabular}{|c|c|c|c|c|c|c|c|c|}
\hline & A & $B$ & C & D & $E$ & $\mathrm{~F}$ & G & $\mathrm{H}$ \\
\hline 1 & \multirow{3}{*}{\multicolumn{3}{|c|}{$\begin{array}{l}\text { SOURCE TABLE FOR THE } \\
\text { NUMERIC CALCULATION OF } \\
\text { THE WORK }\end{array}$}} & & & & & \\
\hline 2 & & & & & & & & \\
\hline 3 & & & & & & & & \\
\hline 4 & No. & V [litres] & $\mathrm{p}[\mathrm{Pa}]$ & & & & & \\
\hline 5 & 1 & 2.0000 & 649531.25 & & & & & \\
\hline 6 & 2 & 2.0040 & 648233.49 & & & & & \\
\hline 7 & 3 & 2.0080 & 646940.90 & & & & & \\
\hline 8 & 4 & 2.0120 & 645653.45 & & & & & \\
\hline 9 & 5 & 2.0160 & 644371.12 & & & & & \\
\hline 10 & 6 & 2.0200 & 643093.87 & & & & & \\
\hline 11 & 7 & 2.0240 & 641821.68 & & & & & \\
\hline 12 & 8 & 2.0280 & 640554.51 & & & & & \\
\hline 13 & 9 & 2.0320 & 639292.33 & & & & & \\
\hline 14 & 10 & 2.0360 & 638035.12 & & & & & \\
\hline 15 & 11 & 2.0400 & 636782.84 & & & & & \\
\hline 16 & 12 & 2.0440 & 635535.47 & & & & & \\
\hline 17 & 13 & 2.0480 & 634292.98 & & & & & \\
\hline 18 & 14 & 2.0521 & 633055.34 & & & & & \\
\hline 19 & 15 & 2.0561 & 631822.51 & & & & & \\
\hline 20 & 16 & 2.0601 & 630594.48 & & & & & \\
\hline 21 & 17 & 2.0641 & 629371.21 & & & & & \\
\hline 22 & 18 & 2.0681 & 628152.68 & & & & & \\
\hline 23 & 19 & 2.0721 & 626938.86 & & & & & \\
\hline 24 & 20 & 2.0761 & 625729.72 & & & & & \\
\hline
\end{tabular}

Figure 4. The source table for the numerical calculation of the work.

In column A of the table, we generated an increasing series with a unit step from 1 to $n=1000$. In column $\mathrm{B}$, we have generated the values of the volume from the start value $V_{l}$ with the help of the series from column A, the volume quantum being determined by the number of divisions $n$ in which the interval $\left[V_{1}, V_{2}\right]$ is divided. In column $\mathrm{C}$, we have calculated the pressure corresponding to each value of the volume from column $\mathrm{B}$ utilizing 
the equation of state. For the calculations, we have introduced the following names of cells and domains: "Pressure_1" for cell B13, "Pressure _2" for cell B14, "Delta_V" for cell B20 in the main spreadsheet, and "Domain_P" for the domain in which we calculate the values of the pressure in column $\mathrm{C}$ of the table from the secondary sheet. Applying the trapezoidal method for the numerical evaluation of a defined integral we have written in cell B21 of the main sheet the following Excel formula:

"= $(\text { SUM }(\text { Domain_P })-(1 / 2) *(\text { Pressure_1 }+ \text { Pressure_2 }))^{*}$ Delta_V".

It can be observed that the calculated value, $L^{*}$, in cell B21 is approximately equal to the calculated value, $L$, in cell B15. In cell B23, we have calculated the relative error at the numerical calculation of the work, as compared to the value from cell B15 of the work calculated analytically.

Modifying the number of divisions $n$ of the interval $\left[V_{l}, V_{2}\right]$ and correspondingly redrawing the source table, it can be observed the effect on the result displayed in cell B21. It is thus verified that with the growth of $n$, the value of the work from cell B21 tends towards the value of the work from cell B15.

Figure 5 presents the main spreadsheet of the toold designed for the comparative analysis between an isothermal process and an adiabatic one, both characterized by the same initial state. This tool can be obtained by reorganizing the spreadsheets of the tool previously presented for the analysis of the isothermal process. Thus, the main spreadsheet rendered in Figure 3 is reorganized as shown in Figure 5. The input data are filled in with the adiabatic coefficient, $\gamma$, whose value is introduced in cell B5 and the section "Results" comprises the subsection displaying the values calculated for the initial pressure, $p_{1}$, the final pressure, $p_{2}$, the final temperature, $T_{2}$, and the work, $L$, for the isothermal process in column B and the subsection displaying the values calculated for the same measures of the adiabatic process in column $C$.

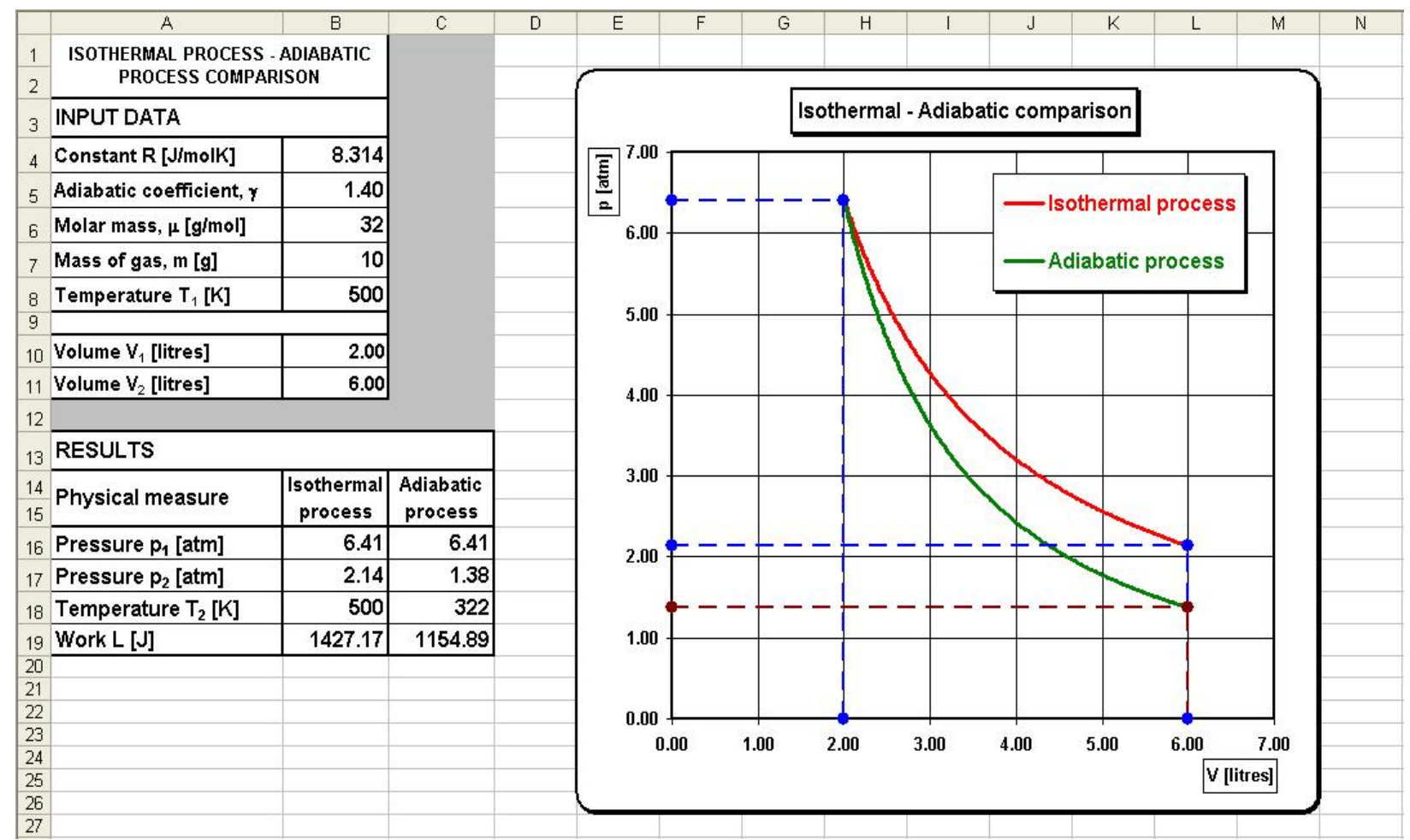

Figure 5. The main spreadsheet of the tool intended for the comparative analysis between the isothermal process and the adiabatic process. 
For the calculations and the graphic representation, we have taken into account both the equation of the isothermal process and the equation of the adiabatic process for an ideal gas.

Next to the tables with the input data and the results, we have placed the graph which overlaps the adiabat curve with the isothermal curve in the coordinate's pressure-volume. On this graph, there are highlighted the initial common state for the isothermal and adiabatic processes, and the final states for the two processes. The source table of the graph has been placed in a secondary spreadsheet of the Excel file. By modifying the input data, we can trace how the results are modified and the isothermal and adiabatic curves change in relation to each other.

\section{Conclusions}

Student participation in the development of the didactic tools described in this paper can be beneficial for the understanding of the model of the ideal gas and the isothermal transformation. The classroom use of the three tools constitutes a conclusive example of highlighting the features of the Excel program.

With the help of the first tool, students can verify the Boyle-Mariotte law of the ideal gas and they can confront the theoretic model against the experimental data. Also, students can explore the graph of the tool to determine the atmospheric pressure and they can compare the value obtained from the calculation to the value obtained from a direct measurement with the barometer in the physics laboratory. Students can therefore find out that the mathematic model can be improved acknowledging the difference between the knowledge of nature and the knowledge of physical models.

With the second tool students can easily understand the isothermal transformation of the ideal gas with the help of the pressure-volume graph and they can clarify certain aspects related to the concept of mechanical work in thermodynamics. Therefore, this tool offers students an alternative demonstration for the calculation relation of the work in the isothermal transformation of the ideal gas. This demonstration, based on the geometrical interpretation of the work within the pressure-volume graph, utilizes the capacity to manipulate the data from the spreadsheet. The numeric calculation of the work constitutes an important step towards the assimilation of a key concept of mathematical analysis, namely, the concept of definite integral. Students can have a better grasp of the way in which knowledge is transferred between physics and mathematics.

With the third tool, students can observe the difference between an isothermal process and an adiabatic process represented graphically in pressure-volume coordinates when both processes are characterized by the same initial state. This tool can be developed from the previous tool by reorganizing spreadsheets. The possibility that students have to transform an Excel tool in another Excel tool to simulate a physical phenomenon or to solve a problem can be employed as an efficient component in the evaluation process.

The tools presented allow the comparative study of a great number of situations using various sets of input data to trace the feedback in results. Students also have the opportunity to start, if not having done so already, devising a procedure to draw graphs according to mathematical relations and/or according to the data obtained following the measurements from the laboratory. The effects produced by the changes in the parameters to certain phenomena prompt students to establish correlations between parameters, to interprete them using the graphs obtained, motivating them to search for new situations. Employing Excel spreadsheets can draw students closer to the study of Physics, so that they participate in the study proposed by this technique. 


\section{References}

Argesanu, L. R. (2011). Experimental physics works for lyceum. Bucharest: Didactic and Pedagogic Publisher.

Caretto, L., McDaniel, D., \& Mincer, T. (2005). Spreadsheet calculations of thermodynamic properties (Paper 2005-2588). In Proceedings of the 2005 American Society for Engineering Education Annual Conference \& Exposition Copyright.

Clarkson, S., Mills, P., Sweeney, W. V., \& Marino, R. (2000). A new approach to teaching introductory science: The gas module. J. Chem. Educ., 77(9), 1161.

Grigore, I., Miron, C., \& Barna, E. S. (2014, October 24-25). Using Excel spreadsheets to process data in physics didactic experiments (pp. 454-459). In Proceedings of the 9th International Conference on Virtual Learning, Bucharest.

Grigore, I., Miron, C., \& Barna, E. S. (2015a). Using Microsoft Excel in teaching and learning relativistic kinematics. Romanian Reports in Physics, 67(2), 716-732.

Grigore, I., Miron, C., \& Barna, E. S. (2015b, October 31). Exploring Excel spreadsheets in the teaching and learning of certain concepts of statistical physics and thermodynamics (pp. 399-405). In Proceedings of the 10th International Conference on Virtual Learning, Timisoara.

Lewis, D. L. (1997). A simple Boyle's law experiment. J. Chem. Educ., 74(2), 209.

Liu, Y. (2011). Development of instructional courseware in thermodynamics education. Computer Applications in Engineering Education, 19, 115-124.

Loverude, M. E., Kautz, C. H., \& Heron, P. R. L. (2002). Student understanding of the first law of thermodynamics: Relating work to the adiabatic compression of an ideal gas. American Journal of Physics, 70(2), 137-148.

Lwin, Y. (2000). Chemical equilibrium by Gibbs energy minimization on spreadsheets. Int. J. Engng Ed., 16(4), 335-339.

Richmond, T. G., \& Parr A. (1997). A U-tube experiment to discover the curve in Boyle's law. J. Chem. Educ., 74(4), 414.

Sandler, S. I. (1997). Spreadsheets for thermodynamics instruction: Another point of view. Chemical Engineering Education (C.E.E.), 31(1), 18-20.

Serway, R. A., \& Jewett, J. W. Jr. (2013). Physics for scientists and engineers with modern physics (9th ed.). Thomson Brooks/Cole. ISBN-13: 978-1-133-95405-7.

Sinex, S. A. (2008). The Boyles law simulator: A dynamic interactive visualization for discovery learning of experimental error analysis. Spreadsheets in Education (eJSiE), 3(1), Article 2.

Stammitti, A. (2013, April). Spreadsheets for assisting transport phenomena laboratory experiences. Education for Chemical Engineers, 8(2), e58-e71. 